\title{
An efficient method to generate random distribution of fibres in continuous fibre reinforced composites
}

DOI:

$10.1002 /$ pc. 25344

Document Version

Accepted author manuscript

Link to publication record in Manchester Research Explorer

\section{Citation for published version (APA):}

Ge, W., Wang, L., Sun, Y., \& Liu, X. (2019). An efficient method to generate random distribution of fibres in continuous fibre reinforced composites. Polymer Composites, 40(12), 4763-4770. https://doi.org/10.1002/pc.25344

\section{Published in:}

Polymer Composites

\section{Citing this paper}

Please note that where the full-text provided on Manchester Research Explorer is the Author Accepted Manuscript or Proof version this may differ from the final Published version. If citing, it is advised that you check and use the publisher's definitive version.

\section{General rights}

Copyright and moral rights for the publications made accessible in the Research Explorer are retained by the authors and/or other copyright owners and it is a condition of accessing publications that users recognise and abide by the legal requirements associated with these rights.

\section{Takedown policy}

If you believe that this document breaches copyright please refer to the University of Manchester's Takedown Procedures [http://man.ac.uk/04Y6Bo] or contact uml.scholarlycommunications@manchester.ac.uk providing relevant details, so we can investigate your claim.

\section{OPEN ACCESS}




\title{
An efficient method to generate random distribution of fibres in continuous fibre reinforced composites
}

\author{
Weijian Ge ${ }^{\mathrm{a}}$, Libo Wang ${ }^{\mathrm{b}}$, Yongle Sun ${ }^{\mathrm{c}}$, Xuqing Liu ${ }^{\mathrm{d}, *}$ \\ ${ }^{a}$ Department of Aeronautics, Imperial College London, South Kensington Campus SW7 2AZ, UK \\ ${ }^{b}$ School of Material Science and Engineering, Henan Polytechnic University, Jiaozuo Henan, 454000, China \\ ${ }^{c}$ School of Mechanical, Aerospace and Civil Engineering, University of Manchester Manchester M13 9PL, UK \\ ${ }^{d}$ School of Materials, University of Manchester Manchester M13 9PL, UK
}

\begin{abstract}
This paper presents a new method based on iterative improvement to generate the random microstructure of continuous fibre reinforced composite with high fibre volume fraction (up to 70\%), overcoming the jamming limit of the traditional Random Sequential Adsorption(RSA) method. The novelty of the proposed method lies in an iterative improvement of the generated microstructure through separating initially randomly set positions of the fibres. Without complex heuristic steps, this algorithm can generate different fibre distributions with fast speed and great simplicity. The offset magnitude of the separation process is adjusted according to fibre volume fractions to speed up the generation. Statistical analysis is then performed on the generated fibre distribution. Five descriptors including Voronoi Polygon Area, Nearest Neighbour Distances, Nearest Neighbour Orientation, Ripley's K Function and Pair Distribution Function are used to compare the proposed algorithm and the RSA with completely spatial random (CSR) distribution for both short and far distances. Fibre distributions generated by this new algorithm are proved to have good randomness. An FEA example is also presented to predict the effective elastic property of the carbon fibre epoxy composites, and a reasonable agreement with the experimental result is achieved. The proposed algorithm provides a useful tool to generate micromechanical models that can be used to predict and understand the mechanical behaviour of fibre reinforced composites.
\end{abstract}

Keywords: microstructure, statistics, elastic properties, random representative volume element

\section{Introduction}

Fibre reinforced composites (FRC), due to their high specific strength and stiffness, are widely used in many different industries such as aeronautics, automobile, maritime etc[1]. In the structure design process, it is important to obtain accurate properties of the composite material. Experimental methods are the most traditional and important ways to achieve this, which however will cost a large number of resources. The prediction methods based on micromechanical analysis appear as a good alternative. Thanks to the development of computing technology, the mainstream of predictions are based on one of the numerical methods called finite element analysis with a representative volume element (RVE). Therefore, the generation of the RVE model for FRC has been recognised as a valuable topic to research. The RVE is normally built as a rectangle or square area, the size of which can neither be too large nor too small. A large RVE requires much time and resource for computation, which is not economical or even possible to calculate. While a small RVE could not be representative to reflect the macroscopic property of the composite. Upon this issue, Trais et al.[2] reported the minimum size $\delta$ should be $50 \times$ fibre radius when fibre volume fraction is over $50 \%$.

\footnotetext{
${ }^{*}$ Corresponding author. Tel: $++44(0) 1613064125$

Email address: xuqing. liu@manchester.ac.uk (Xuqing Liu)
}

The spatial arrangement of fibre is also an important issue to affect the mechanical property of the composite. In the early day's analysis of composites material, the spatial arrangement usually was considered as regular and periodic for simplicity. However, such models with regular spatial arrangement suffer many limitations. Trias et al.[3] compared the stress/strain distributions of periodic and real spatial models under a transverse tensile load. He found that periodic models predicted poorly on properties related to damage and failure. Gusev et al.[4] pointed out the randomness of the composite material microstructure had a great influence on the transverse composite elastic constants. Pyrz[5] concluded that in polymer matrix composite, the type of spatial pattern had a significant impact on the overall failure stress. Therefore, for more accurate predictions, the micromechanical model should take into account the stochastic nature of the real fibre distribution.

The actual spatial distribution is very different from periodic and highly depends on the manufacturing process[6]. To capture the irregular spatial distribution of fibres within a composite microstructure, the general approach is to develop a statistically equivalent RVE (SERVE). A SERVE is the smallest region of a generated microstructure which exhibits the same effective stress-strain behaviour as the overall composite[7]. Random sequential adsorption(RSA) method, also called hard-core method is the most direct way to generate SERVE. In this method, by searching one disk after another is randomly positioned into 
a position without overlapping with any previous disks until the coverage is satisfied. However, due to its poor space utilisation, RSA is subjected to a relatively low jamming limit. For circular disks, the jamming occurs around coverage of 54.7\%[8] which is lower than the maximum volume fraction of typically achievable in fibre composites. Therefore, many new approaches have been developed for higher fibre volume fraction.

Previous methods generally can be classed into two types. The first type is to generate a new distribution by perturbations of the original RSA or periodic configuration. Wongsto[9] proposed an algorithm that generates microstructure by disturbing an initially hexagonal array. A.R.Melro et al.[6] developed a faster method by processing two heuristics after the RSA model. Zhang et al.[10] âs method disturbed the initial periodic distribution by the eccentric collision algorithm. Zhu et al.[11] invented a new perturbation algorithm initialised with a model where fibres tangent to each other. These types of methods have problems such as time-consuming and complex to realise. The other type of methods can be named as random sequential expansion(RSE), such as Yang et al.[12]. The main idea is to generate the distribution from a central area and expands one by one until filling the overall area. This type of methods has a great advantage since it can control the process of fibre generation from the beginning to the end so that it is very suitable to perform statistical reconstruction, which refers to generating distribution with the same geometric feature as the experimental samples. McCarthy et al.[13] conducted combined experimental-numerical research and proposed the nearest neighbour algorithm (NNA), generating the distribution based on the nearest neighbour distribution of the samples. Later on, Wang et al.[14] improved the NNA algorithm by introducing the probability equation and the frequency updating equation so that it enabled a closer representation of the realistic fibre distribution. Still, some disadvantages exist such as hard to guarantee the same appearance probability everywhere in the RVE, the fibres gather in the centre area as the RVE window is not fully filled.

All methods mentioned above discarded any situations of overlapping throughout the generation process, which however rejected many potential solutions in the search spaces. Instead, if we start from an arbitrary spatial arrangement and improve the overlappings gradually, it will take less time to find a solution. Following this idea, we provide a new method to generate random fibre distribution based on the iterative improvement algorithm. By setting parameters optimally, this new algorithm is proved to be very efficient to generate spatial arrangement with high fibre volume fraction(up to $70 \%$ ). The algorithm is presented in section 2 and the microstructures generated are analysed with statistic descriptors to demonstrate the randomness in section 3. Finally, as an example, the finite element analysis is implemented to predict the elastic properties of the generated RVE, and the result is compared with the experimental data to show the efficacy of the model in section 4 .

\section{Algorithm Development}

Before we proceed to present the algorithm, three assumptions were made on the microstructure: (1) the object window is considered as a square area $\left(a^{2}\right) ;(2)$ the fibre as circular disks with uniform radius $r_{f}$; (3) the interface area between matrix and fibre is ignored. Therefore, the task can be seen as generating numbers of circles with a coverage of $\phi_{f}$ inside a square area, and the distribution of circles should have high-quality randomness so that it can represent the real situation in the FRC.

This new algorithm solves the task by iterative improvements, the general idea of which can be summarised as (1) define the initial state and the objective state (2) update the state iteratively until it reaches the objective state. The initial state of this algorithm is set to be a number of completely spatial random positioning circles. In the objective state, none intersection between circles exists and the coverage $\phi_{f}$ should be satisfied, which can be expressed as Eq.1

$$
\begin{aligned}
& \sqrt{\left(x_{i}-x_{j}\right)^{2}+\left(y_{i}-y_{j}\right)^{2}} \geq 2 r_{f}+l_{\text {min }} \forall i, j \in N i \neq j \\
& \sum_{i=1}^{N} A_{i}=\phi_{f} a^{2}
\end{aligned}
$$

Where, $x_{i}, y_{i}$ are the position coordinates, $l_{\min }$ is the minimum distance between fibres which can be adjusted to change the fibre distribution and mitigate mesh difficulties of the FEA in case of high fibre volume fraction. Numerical experiment shows, in our algorithm it would be more efficient to separate numbers of circles at once rather than introducing and separating them one by one. Therefore, the number of fibre should be estimated by Eq. 2 at the beginning. Since some circles have very little contribution to the overall coverage such as those near edge as illustrated in Fig.1, the actual number of circles must be larger than our initial estimation. Therefore, more circles need to be introduced during the generation process.

$$
N^{*}=\left[\left(\phi_{f} a^{2}\right) /\left(\pi r_{f}^{2}\right)\right]
$$

The core part of the algorithm is how to improve the state. In the generation process, there will be many situations of overlaps among different states. Some will be better than others, which are closer to the objective state. Similarly as the idea of genetic computing, 'mutation' can applied by random movement of each circle, and a test should be set to compute whether the result is 'better' or 'worse' than the original state. If it is worse, we discard it; if it is better, we keep it, and that becomes the new original. For this problem, however, since we already know moving the circles away from each other will always be 'better' if they overlap. Therefore, we can design the algorithm as Alg.1, where we check every circle position with other circles, and offset them if there is any overlap. Gradually, the number of overlap will decrease and finally reduce to 0 as illustrated in Fig.2. Then we can check the coverage, if not satisfied, extra fibre will be added. The detailed offset process is acheived as 


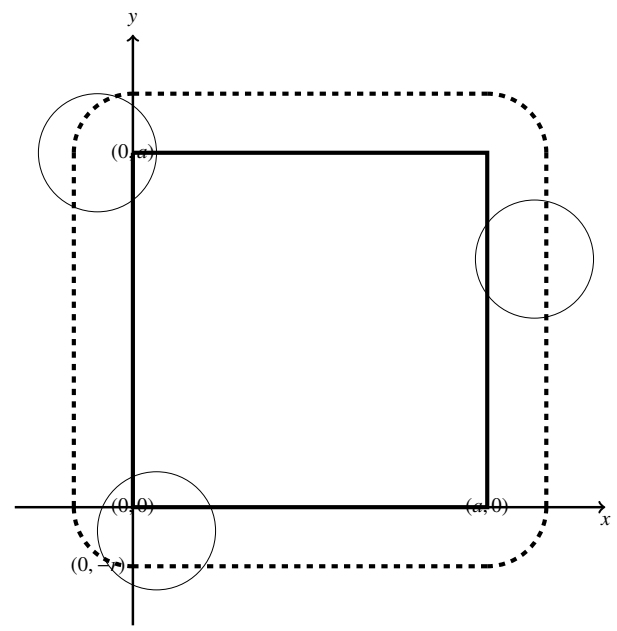

Figure 1: An illustration of RVE area, the square with solid line is the RVE window, and circles whose centre points are inside the dashed line will contribute to the total volume fraction

Eq.3.

$$
\begin{aligned}
& x_{1}=x_{1}+\frac{x_{1}-x_{2}}{\left|x_{1}-x_{2}\right|} \cdot w \cdot \operatorname{random}(0,1) \\
& y_{1}=y_{1}+\frac{y_{1}-y_{2}}{\left|y_{1}-y_{2}\right|} \cdot w \cdot \operatorname{random}(0,1)
\end{aligned}
$$

Where $w$ is the magnitude of the offset in the separation process. The model would have quicker converge and better randomness if this value is optimally set. After a trial and error process, an empirical function for setting of $w$ is found as Eq.4

$$
w=-11.5 \phi_{f}^{2}-4.3 \phi_{f}+8.5
$$

In order to illustrate the effect of $w$ adjustment, we compared running times of algorithms with adjusted and fixed $w .8$ different $\delta=50 \mathrm{RVE}$, with $\phi_{f}$ ranging from $45 \%$ to $65 \%$ were generated in this comparison, and each had 20 realisations. The Fig. 3 presents the average running time, and error bars are included to show standard derivations. We can conclude that the running time decreases dramatically when $w$ is optimally set, especially for large volume fraction. Meanwhile using RVE with $\delta=50$ and $\phi_{f}=65 \%$ as a benchwork, we compared the speed among different algorithms introduced in the literature. The results are presented as Tab.1, from which we can see though neat and simple, the proposed algorithm is faster than any other methods.

Table 1: The reported average running time for $\delta=50, \phi_{f}=0.65$

\begin{tabular}{lc}
\hline Method & Time \\
\hline Current Algorithm & $1.75 \mathrm{~min}$ \\
M.V. Pathan[15] & $107.02 \mathrm{~min}$ \\
RAND $_{u} S T R U_{G} E N[6]$ & $3.31 \mathrm{~min}$ \\
Wonggsto and Li[9] & $7.20 \mathrm{~min}$ \\
Trias [3] & $1145.09 \mathrm{~min}$ \\
\hline
\end{tabular}

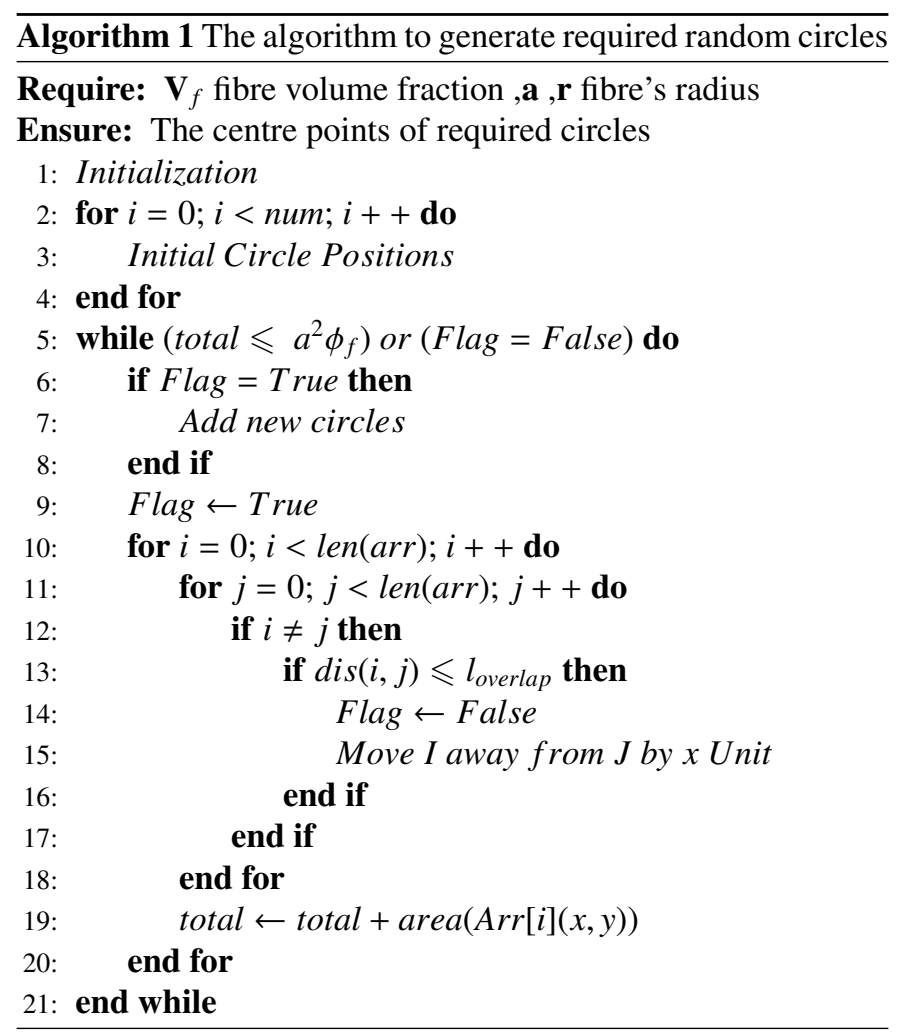

\section{Statistical characterisation of the spatial distribution}

In order to quantitatively characterise the randomness of fibre spatial distribution within the generated RVE, 5 different statistical descriptors are introduced.including Voronoi polygon area, nearest neighbour distances, nearest neighbour orientation, Ripley's K Function, and Pair Distribution Function.

\subsection{Voronoi polygon area}

The 2D Voronoi tessellation is a subdivision of region, determined by a set of points, where each point has associated with it a sub-region that is closer to it than to any other. The standard deviation of the areas of the Voronoi polygons defines the more or less periodic distribution of the circles. For example, in a periodic arrangement of circles, each of the Voronoi cell will have the identical area, so the deviation equals to 0 . Therefore, we can use the coefficient of variation which defines as Eq.5 to compare the randomness of the distribution.

$$
C_{v}=\sigma / \mu
$$

Where $\sigma$ and $\mu$ are mean and standard deviation respectively. We extracted this coefficient for a generated microstructures with $\delta=50$ and $\phi_{f}=65 \%$, compared to those reported by Pathan.[15], and listed in Table 2. At such volume fraction, the proposed algorithm achieves a relatively high coefficient of variation compared with other algorithms, indicating decent randomness of the generated microstructures. 

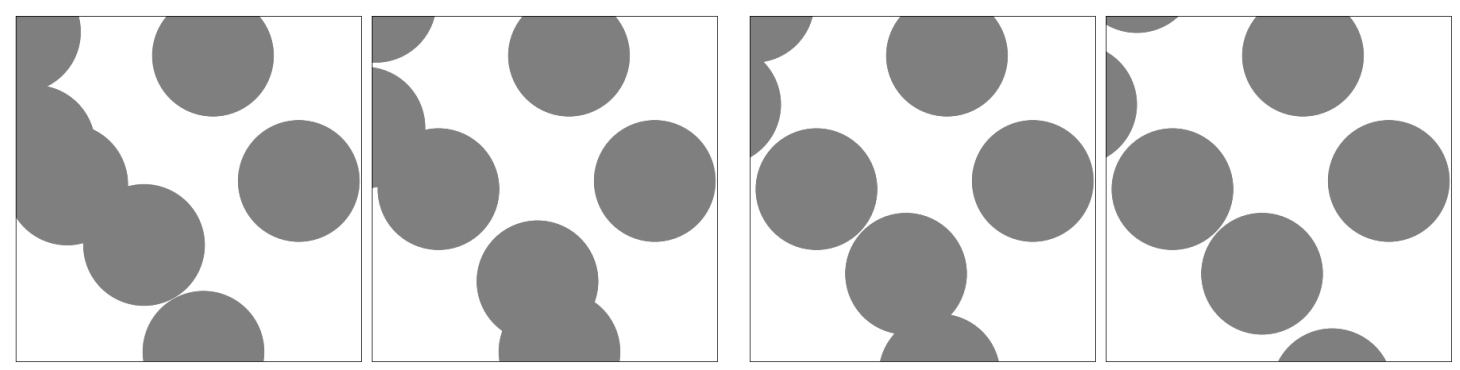

Figure 2: An example of the spatial distribution of circles at initial state, 2 successive iterations and the final state

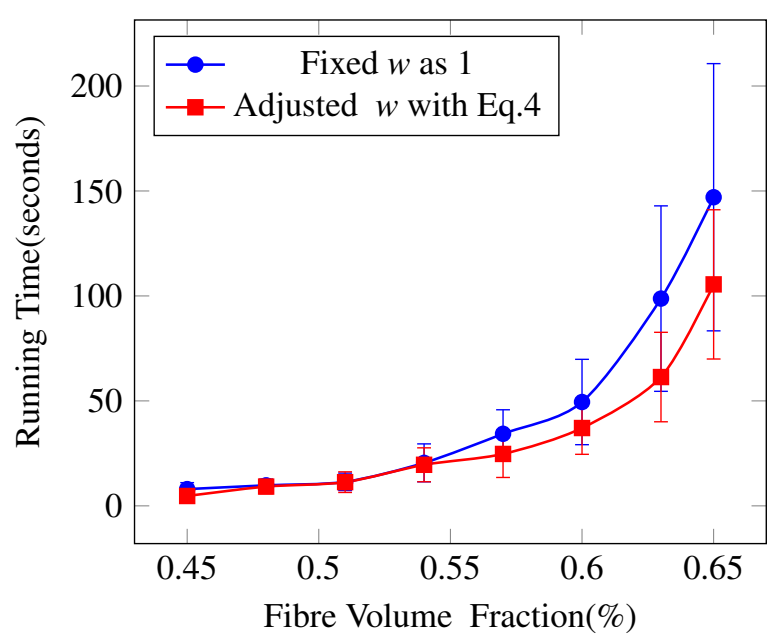

Figure 3: The running times of algorithm with adjusted and fixed $w$ for 8 different $\phi_{f}$ for $\delta=50 \mathrm{RVE}$

Table 2: The reported average for $\delta=50, \phi_{f}=0.65$

\begin{tabular}{lc}
\hline Method & $C_{v}$ \\
\hline Current Algorithm & 0.110 \\
M.V. Pathan[15] & 0.114 \\
RAND $_{u} S T R U_{G} E N[6]$ & 0.099 \\
Wonggsto and Li[9] & 0.077 \\
\hline
\end{tabular}

\subsection{Nearest Neighbour Distances}

Nearest neighbour distances are always measured in Probability Density Function(PDF) of the distance from one typical point to the its nearest neighbours. They provide information on the short distance fibre interaction and indicate whether the point pattern shows some degree of clustering. For example, if the PDF plot shows a peak followed by a dramatic decrease, this could indicate the clustering of the fibres, otherwise it is caused by high fibre volume fraction if the 2nd or 3rd neighbour distances PDF plot show a smooth decrease. This is one of the most important descriptors, since Hojo et al.[16] found that it had a crucial effect on the damage evolution in the fibre/matrix interface, which influences the consequential failure behaviour of the composites. Damage is more likely to initiate in the clustering area. The study of nearest neighbour distances of this algorithm including the first nearest neighbour and second nearest neighbour distances. Choosing RVE with $\sigma=50$ and $\phi_{f}=50 \%$, we compared the performance of distribution generated by this algorithm and RSA method. The Fig.5 and Fig. 6 present the average PDF of the first and second nearest neighbour distance respectively, with error bars showing the standard deviations. From these two graphs, we can see the distribution of neighbour distances in this algorithm is very similar to that of the RSA, which shows this algorithm can achieve a good randomness of distribution.

\subsection{Nearest Neighbour Orientations}

The nearest neighbour orientation can be determined easily given the first nearest neighbour position. The orientation here is measured anti-clockwise in respect of the horizontal axis. Different from the nearest neighbour distances, this one is presented as a Cumulative Distribution Function(CDF). If the distribution of the fibres is a perfectly random distribution, the CDF curve will be a straight diagonal line, which means each orientation has the same probability of occurring. Deviations from this line will indicate some orientations are preferred. The periodic distribution will then have a stair shape curve.

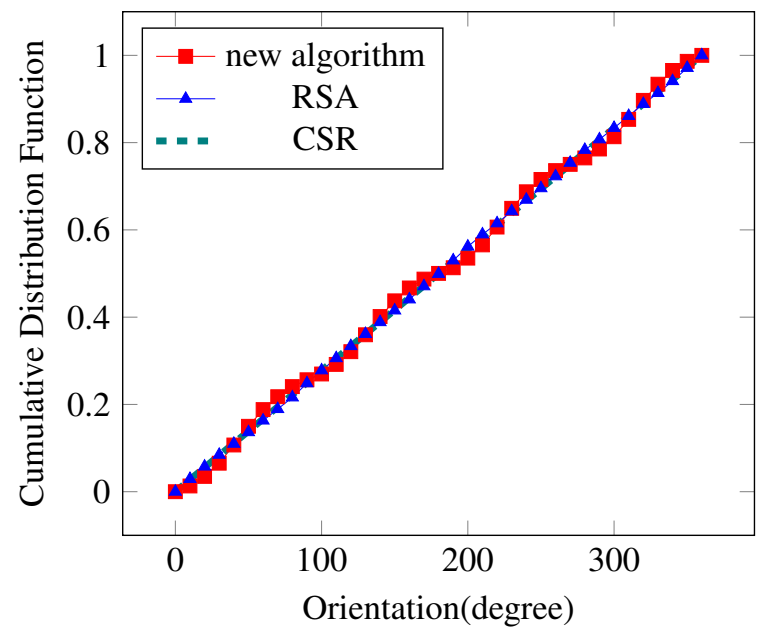

Figure 7: The orientation CPD for RVE with $\delta=50$ and $\phi_{f}=0.5$

Similarly, we compared the performance of this algorithm and the RSA. Fig.7 shows both two curves are very close to the straight line which indicates completely spatial random distribution. Such comparison shows this algorithm is able to capture the orientation randomness of fibres distribution in composites. 

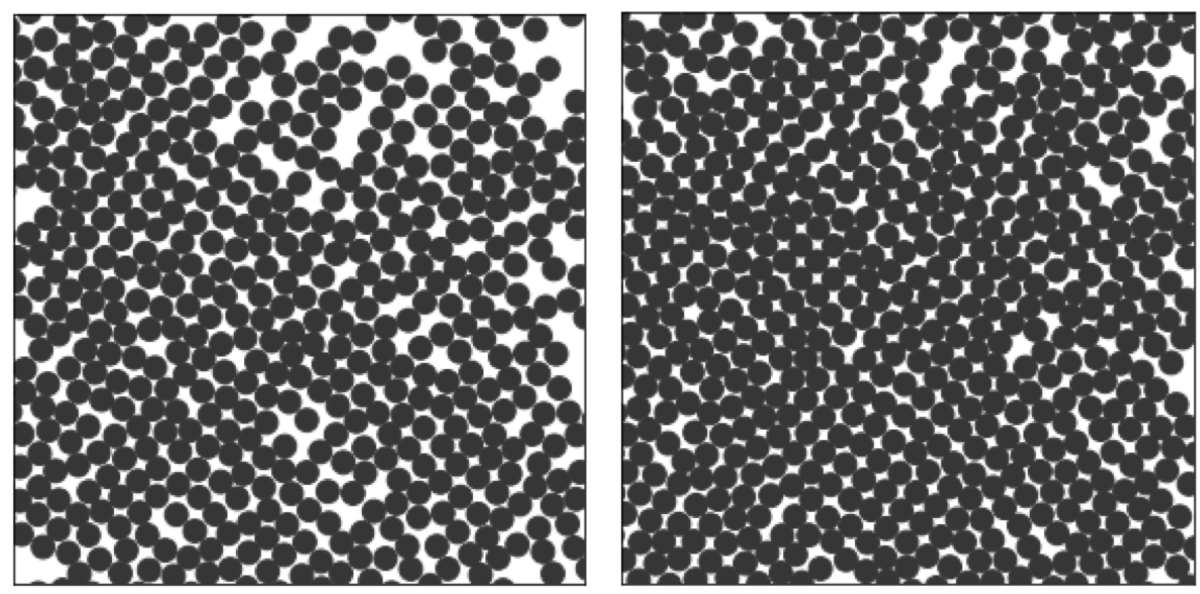

Figure 4: Two examples of $\delta=50$ RVEs with $60 \%$ and $70 \%$ coverage respectively

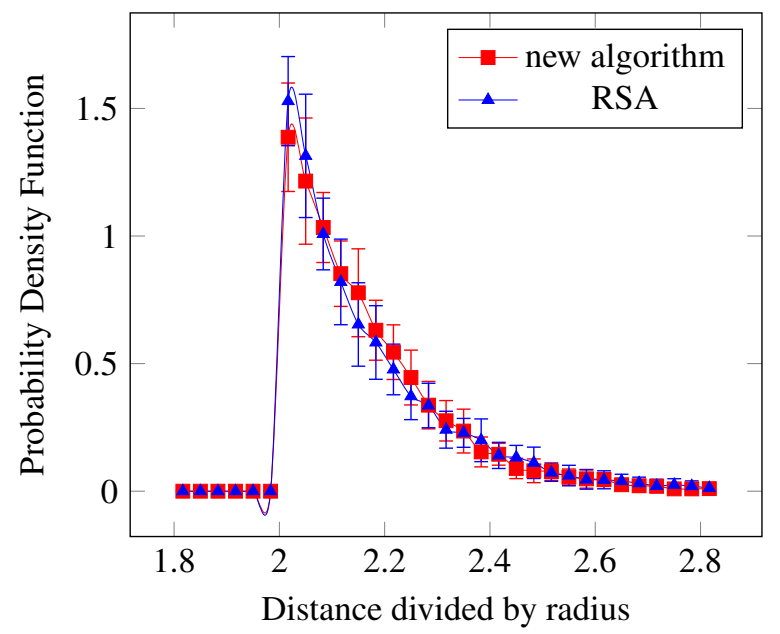

Figure 5: First nearest neighbour distance PDF for RVE with $\delta=50$ and $\phi_{f}=$ 0.5

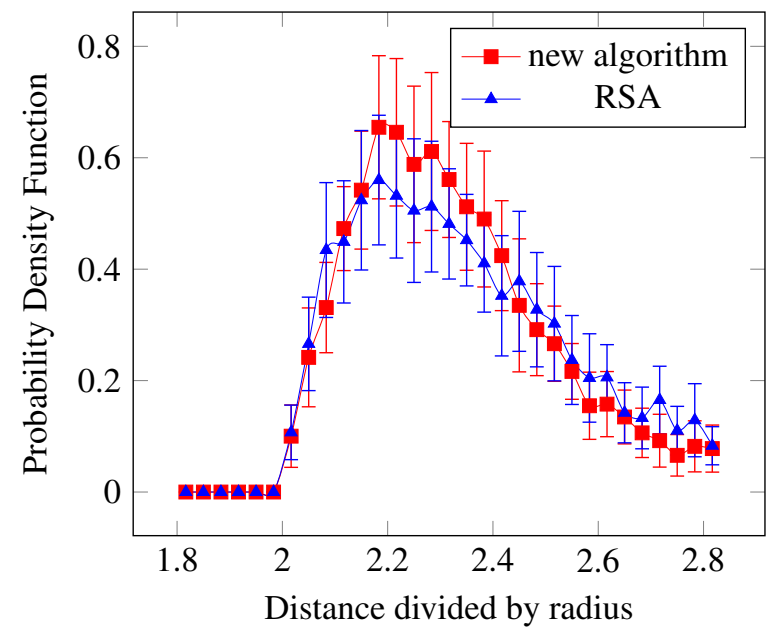

Figure 6: Second nearest neighbour distance PDF

\subsection{Ripley's K Function}

Ripley's $\mathrm{K}$ function, also known as second-order intensity function, has been regarded as one of the most informative descriptors of spatial pattern [5] which concerns the long distance interaction of inclusion. It is defined as the ratio between the number of additional points within a radial distance $r$ of an arbitrary points against the number of points per unit area. Correlated for the edge effects, the Ripley's K function can be calculated as:

$$
K(h)=\frac{A}{N^{2}} \sum_{i} \sum_{j \neq i} \frac{I\left(d_{i j} \leq h\right)}{w\left(l_{i}, l_{j}\right)}
$$

Where $\mathrm{A}$ is the area of RVE window, $\mathrm{N}$ refer to the number of fibres in the windows, $d_{i j}$ is the distance between two points $i$ and $j, \mathrm{I}$ is an indicator which equal to 1 if the condition in the bracket holds true, otherwise equal to $0 . w\left(l_{i}, l_{j}\right)$ denotes the proportion of circumference of the circle with $d_{i j}$ as radius laying inside the window. For a completely random distribution, it is easy to derivate that Ripley's K function: $K(h)=\pi h^{2}$. Above the Completely Spatial Random (CSR) pattern plot, indicate clusterings in the distribution, otherwise, shows some degree of regularity. A Stair-shaped plot exhibit the pattern has periodicity such as square or hexagonal distribution [6]. It can be seen from the Fig.8, both this algorithm and the RSA perfectly agreed with the CSR curve $\left(K=\pi r^{2}\right)$, only having a little deviation at short distances. This indicates that generated distribution conformed well to a CSR distribution, especially at long distances.

\subsection{Pair Distribution Function}

According to Ripley's K function, the pair function curve can also be plotted as the figure 9. The curve is finally converge into 1 , which again proves the distribution generated with this evolutionary algorithm largely conform to the CSR. Therefore, this algorithm is proved to be capable of generating highly random fibre distribution, which provides a powerful tool for simulation with limited experimental data of fibre distribution. 


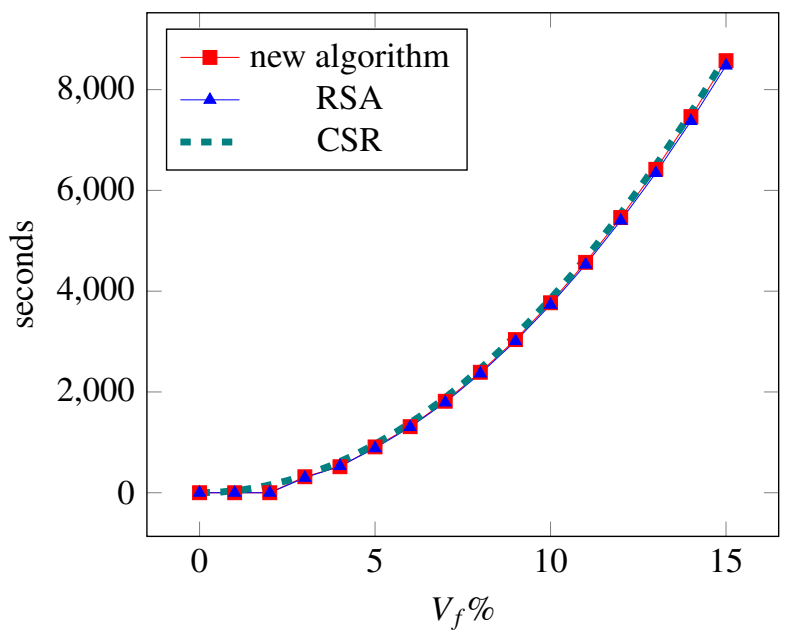

Figure 8: The Ripley's function for RVEs with $\delta=50$ and $\phi_{f}=0.5$

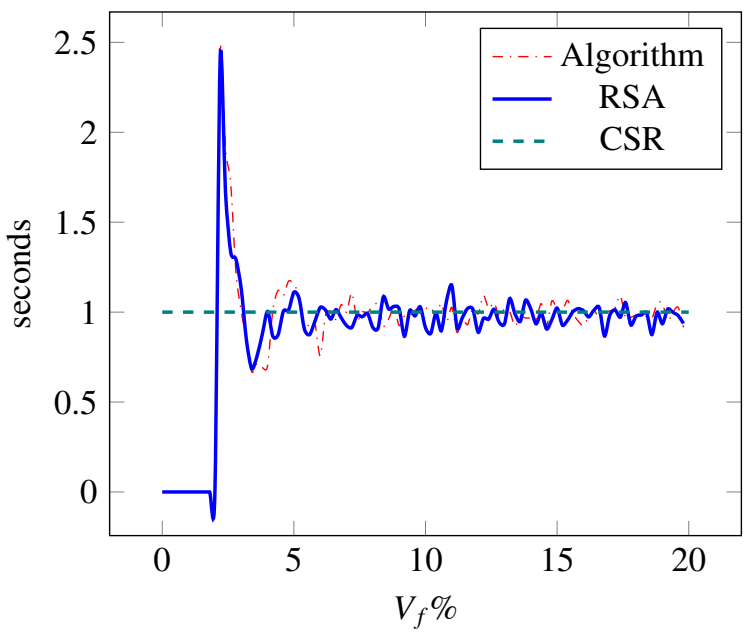

Figure 9: Pair distribution function for RVEs with $\delta=50$ and $\phi_{f}=0.5$

The pair distribution reflects the probability of finding an additional point within an annular area with the thickness of $d h$, which can be calculated as: [17]

$$
g(h)=\frac{1}{2 \pi N_{a} h \dot{d} h} \cdot \frac{1}{N} \sum_{i=2}^{N} n_{i}(h)=\frac{1}{2 \pi h} \cdot \frac{d K(h)}{d h}
$$

Pair distribution function contains the same statistical information as the K-function, but is more clear and easier to understand. For CSR pattern, the $g(h)$ have a constant value 1 for all distances considered. In this sense, a statistically valid distribution should oscillate close to 1 as the distance increases. In Fig.9, both curves of this algorithm and RSA have fluctuant at short distances, but tend to oscillate close to 1 as the distance increases, which demonstrate the sufficient randomness of distributions.

\section{Numerical verification}

As a numerical verification, an effective elastic property prediction is presented Table 3. Effective properties of fibre
Table 3: The constituent property of T300,BSL914C laminate

\begin{tabular}{lc|lc}
\hline \multicolumn{2}{l}{ Fibre(T300 Carbon Fibre) } & \multicolumn{2}{l}{ Matrix(BSL914C epoxy) } \\
\hline Property & Value & Property & Value \\
\hline$R f / \mu m$ & 3.5 & E/Gpa & 4.0 \\
$E_{1} / \mathrm{GPa}$ & 230 & $v$ & 0.38 \\
$E_{2} / \mathrm{GPa}$ & 15 & & \\
$G_{12} / \mathrm{GPa}$ & 15 & & \\
$G_{23} / \mathrm{GPa}$ & 7 & & \\
$v_{12}$ & 0.2 & \\
$v_{23}$ & 0.07 & \\
Volume Fraction & $60 \%$ & \\
\hline
\end{tabular}

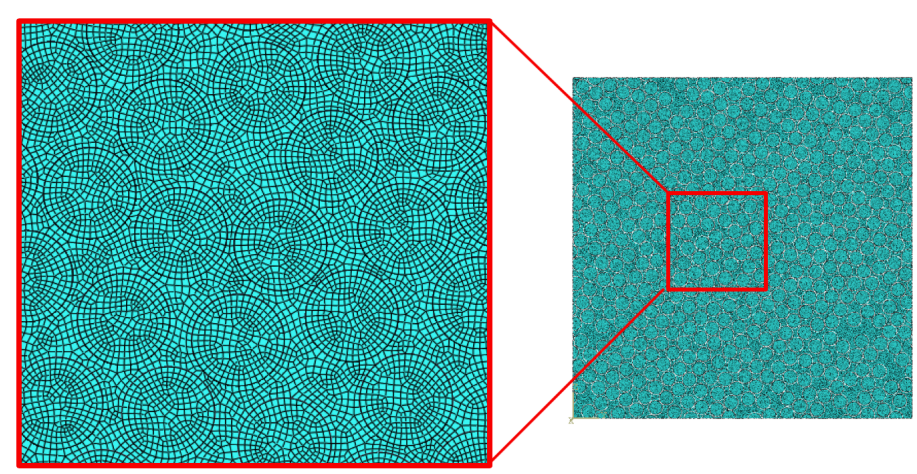

Figure 10: A meshed RVE with $\delta=50$ and $\phi_{f}=60 \%$

tows in a textile composite are generally obtained through micromechanical models based on the fibre volume ratio and constitutive properties of each constituent[18]. In this example, with the help of the algorithm, we can generate an FE model, and carry out a numerically homogenisation. The model of carbon fibre reinforced epoxy composite is chosen as T300BSL/914C is generated. The constituent properties of this composite are given below[19]. A transversely cross-sectional area $175 \times$ 175 with the fibre coverage of $60 \%$ was generated with the new algorithm. The longitudinal length is trivial and set to be 0.5. This RVE was imported into ABAQUS with python scripts and meshed with two types of elements hexahedral elements(C3D8R) and tetrahedral elements (C3D6) for fibre and matrix section respectively. Perfect interface was imposed between fibres and matrix, by tying them together. Totally, about 200000 elements were generated in the RVE as the Fig.10 Based on a volume average approach[20], we can obtain the stiffness matrix $C_{i j}$ as Eq.8

$$
\begin{aligned}
\bar{\sigma}_{i} & =C_{i j} * \bar{\varepsilon}_{j} \\
\bar{\varepsilon} & =\frac{1}{V} \int_{v} \varepsilon_{i j} d v \\
\bar{\sigma} & =\frac{1}{V} \int_{v} \sigma_{i j} d v
\end{aligned}
$$


Table 4: The simulation and experimental result

\begin{tabular}{lcc}
\hline *Elastic Property & FEA & *Experiment \\
\hline$E_{1} / \mathrm{GPa}$ & 135.86 & 138.0 \\
$E_{2} / \mathrm{GPa}$ & 9.838 & 11.0 \\
$v_{12}$ & 0.30 & 0.28 \\
$v_{23}$ & 0.35 & 0.40 \\
$G_{12} / \mathrm{GPa}$ & 4.185 & 5.5 \\
\hline
\end{tabular}

Next, according to Eq.9[10], five engineering constants can therefore be calculated:

$$
\begin{aligned}
& E_{1}=C_{11}-2 C_{12}^{2} /\left(C_{22}+C_{23}\right) \\
& v_{12}=C_{12} /\left(C_{22}+C_{23}\right) \\
& E_{2}=\left[C_{11}\left(C_{22}+C_{23}\right)-2 C_{12}^{2}\right]\left(C_{22}-C_{23}\right) /\left(C_{11} C_{22}-C_{12}^{2}\right) \\
& v_{23}=\left[C_{11} C_{23}-C_{12}^{2}\right] /\left(C_{11} C_{22}-C_{12}^{2}\right) \\
& G_{12}=C_{66}
\end{aligned}
$$

The average values of engineering constants from 20 different realisations are presented in the Table 4, from which we can see the prediction has a good agreement with the experimental results.

\section{Conclusion}

Based on the idea of iterative improvement, a new algorithm has been developed to generate the random spatial distribution for fibres in FRC with up to $70 \%$ fibre volume fraction. Without complex heuristic steps, this algorithm was proved to be faster than most of the previous algorithms introduced in the literature. In addition, evaluated with 5 different statistical descriptors, the distribution generated by this algorithm turned out to have very good randomness. The algorithm was employed to predict numerically the effective elastic properties prediction of unidirectional T300, BSL914C FRC from its constituent properties. The results show a good agreement with the experimental data. This algorithm, therefore, provides a powerful tool to reproduce the microstructure of the composites when the actual spatial information is not available.

\section{References}

[1] Zhenqiang Zhao, Peng Liu, Chunyang Chen, Chao Zhang, and Yulong Li. Modeling the transverse tensile and compressive failure behavior of triaxially braided composites. Composites Science and Technology, 172:96$107,2019$.

[2] Mayugo JA Hurtado JE. Trais D, Costa J. Determination of the critical size of a statistical representative volume element(srve) for carbon reinforced polymers. Acta Metallurgica, 54(13):3471-84, 2006.

[3] Mayugo JA Hurtado JE. Trais D, Costa J. Random models verus periodic models for fibre reinforced composites. Comput Mat Sci, 38(2):316-324, 2006.

[4] Hine P.J Ward I.M Gusev, A.A. Fibre packing and elastic properties of a transversely random unidirectional glass/epoxy composite. Compos.Sci.Technol., 60:535-541, 2000.

[5] R. Pyrz. Quantitative descpition of the microstrcture of composites.part i:morphology of unidirectional composite systems. Compos.Sci.Technol., 50(2):197-208, 1994.
[6] S.T.Pinho A.R.Melro, P.P.Camanho. Generation of random distribution of fibres in long-fibre reinforced composites. Compos.Sci.Technol., 68:2092-2102, 2008.

[7] Pagano NJ. Swaminathan S, Ghosh S. Statistically equivalent representative volume elemets for unidirectional composite microstructures: Part i-without damage. Jounral of Composite Materials, 40(7):583-604, 2006.

[8] S Torquato, OU Uche, and FH Stillinger. Random sequential addition of hard spheres in high euclidean dimensions. Physical Review E, 74(6):061308, 2006.

[9] S.Li A.Wongsto. Micromechanical fe analysis of ud fibre-reinforced composites with fibres distributed at random over the transverse cross-section. Composites Part A, 36:1246-1266, 2005.

[10] Taotao Zhang. A comparison between random model and perodic model for fiber-reinforced composites based on a new method for generating fiber distribution. Polym.Compos., 38:77-86, 2017.

[11] Zhao Liu Chao Zhu, Ping Zhu and Wei Tao. Numerical investigation of fiber random distribution on the mechanical properties of yarn in-plane woven carbon fiber-reinforced composite based on a new pertubation algorithm. Jounral of Composite Materials, 0(1-17), 2017.

[12] Zhiguo Ran Yujia Liu Lei Yang, Ying Yan. A new method for generating random fibre distribution for fibre reinforced composites. Compos.Sci.Technol., 76:14-20, 2012.

[13] Vaughan Ted J. McCarthy, Conor T. A combined experimental-numerical approach for generating statistically equivalent fibre distributions for high strength laminated composite material. Compos.Sci.Technol., 70(2):291297, November 2009.

[14] Chao Zhang Xiaosheng Gao Wenzhi Wang, Yonghui Dai and Meiying Zhao. Micromechanical modelling of fibre-reinforced composites with statitically equivalent random fibre distribution. MDPI, 9(8):624, 2016.

[15] MV Pathan, VL Tagarielli, S Patsias, and PM Baiz-Villafranca. A new algorithm to generate representative volume elements of composites with cylindrical or spherical fillers. Composites Part B: Engineering, 110:267278, 2017.

[16] M.;Hobbiebrunken T.;Adachi T.Tanaka M.;Ha S.K. Hojo, M.; Mizuno. Effect of fiber array irregularities on microscopic interfacial normal stress states of transversly loaded ud-cfrp from viewpoint of failure initiation. Compos.Sci.Technol., 69:1726-1734, 2009.

[17] Nowak Z Ghosh S and Lee K. Tessellation-based computational methods for the charaterization and analysis of heterogenous microstructure. Compos.Sci.Technol., 57(1187-1210), 1997.

[18] Zhenqiang Zhao, Haoyuan Dang, Chao Zhang, Gun Jin Yun, and Yulong Li. A multi-scale modeling framework for impact damage simulation of triaxially braided composites. Composites Part A: Applied Science and Manufacturing, 110:113-125, 2018.

[19] P. Soden. Lamina properties, lay-up configurations and loading conditions for a range of fibre-reinforced composite laminates. Compos.Sci.Technol., 58(7):1011-1022, 1998.

[20] De-ping GAO You-feng LEI, De-ming WEI. Predicting macroscopic effectiveelastic moduli of composites by mirco-mechanics fem. Gas Turbine Experiment and Research, 16(3):11, 2003.

[21] Ever J.Barbero. Finite Element Analysis of Composite Material. CRC Press, 2007. 